Ferrata Storti Foundation

\title{
Iron absorption from supplements is greater with alternate day than with consecutive day dosing in iron-deficient anemic women
}

Haematologica 2020

Volume 105(5):1232-1239

\section{Correspondence:}

NICOLE STOFFEL

nicole.stoffel@hest.ethz.ch

Received: March 1, 2019.

Accepted: August 12, 2019.

Pre-published: August 14, 2019.

doi:10.3324/haematol.2019.220830

Check the online version for the most updated information on this article, online supplements, and information on authorship \& disclosures: www.haematologica.org/content/105/5/1232

(C)2020 Ferrata Storti Foundation

Material published in Haematologica is covered by copyright. All rights are reserved to the Ferrata Storti Foundation. Use of published material is allowed under the following terms and conditions:

https://creativecommons.org/licenses/by-nc/4.0/legalcode. Copies of published material are allowed for personal or internal use. Sharing published material for non-commercial purposes is subject to the following conditions: https://creativecommons.org/licenses/by-nc/4.0/legalcode, sect. 3. Reproducing and sharing published material for commercial purposes is not allowed without permission in writing from the publisher.

\author{
Nicole U. Stoffel, ${ }^{1}$ Christophe Zeder, ${ }^{1}$ Gary M. Brittenham, ${ }^{2}$ Diego Moretti ${ }^{1 *}$ and \\ Michael B. Zimmermann ${ }^{1^{*}}$ \\ ${ }^{1}$ ETH Zurich, Department of Health Science and Technology, Institute of Food, \\ Nutrition and Health, Laboratory of Human Nutrition, Zürich, Switzerland and \\ ${ }^{2}$ Columbia University, Department of Pediatrics, College of Physicians and Surgeons, \\ New York, NY, USA
}

*These authors are co-senior authors

\section{ABSTRACT}

I n iron-depleted women without anemia, oral iron supplements induce an increase in serum hepcidin (SHep) that persists for 24 hours, decreasing iron absorption from supplements given later on the same or next day. Consequently, iron absorption from supplements is highest if iron is given on alternate days. Whether this dosing schedule is also beneficial in women with iron-deficiency anemia (IDA) given high-dose iron supplements is uncertain. The primary objective of this study was to assess whether, in women with IDA, alternate-day administration of 100 and $200 \mathrm{mg}$ iron increases iron absorption compared to consecutive-day iron administration. Secondary objectives were to correlate iron absorption with SHep and iron status parameters. We performed a cross-over iron absorption study in women with IDA ( $\mathrm{n}=19$; median hemoglobin $11.5 \mathrm{mg} / \mathrm{dL}$; mean serum ferritin $10 \mu \mathrm{g} / \mathrm{L}$ ) who received either 100 or $200 \mathrm{mg}$ iron as ferrous sulfate given at $8 \mathrm{AM}$ on days 2,3 and 5 labeled with stable iron isotopes $57 \mathrm{Fe}, 58 \mathrm{Fe}$ and $54 \mathrm{Fe}$; after a 16-day incorporation period, the other labeled dose was given at $8 \mathrm{AM}$ on days 23,24 and 26 (days 2, 3 and 5 of the second period). Iron absorption on days 2 and 3 (consecutive) and day 5 (alternate) was assessed by measuring erythrocyte isotope incorporation. For both doses, SHep was higher on day 3 than on day $2(P<0.001)$ or day $5(P<0.01)$ with no significant difference between days 2 and 5 . Similarly, for both doses, fractional iron absorption (FIA) on days 2 and 5 was $40-50 \%$ higher than on day 3 $(P<0.001)$, while absorption on day 2 did not differ significantly from day 5 . There was no significant difference in the incidence of gastrointestinal side effects comparing the two iron doses $(P=0.105)$. Alternate day dosing of oral iron supplements in anemic women may be preferable because it sharply increases FIA. If needed, to provide the same total amount of iron with alternate day dosing, twice the daily target dose should be given on alternate days, as total iron absorption from a single dose of $200 \mathrm{mg}$ given on alternate days was approximately twice that from $100 \mathrm{mg}$ given on consecutive days $(P<0.001)$. In IDA, even if hepatic hepcidin expression is strongly suppressed by iron deficiency and erythropoietic drive, the intake of oral iron supplements leads to an acute hepcidin increase for 24 hours. The study was funded by ETH Zürich, Switzerland. This study has been registered at www.clinicaltrials.gov as \#NCT03623997.

\section{Introduction}

Anemia affects $~ 33 \%$ of the world population and accounts for $8.8 \%$ of global disability. ${ }^{1}$ Iron deficiency (ID) is considered the most prevalent cause of anemia globally. ${ }^{1}$ In the United States, nearly $10 \%$ of 12 to 49 -year-old females have ID. ${ }^{1,2}$ 
Oral iron supplementation with ferrous sulfate $\left(\mathrm{FeSO}_{4}\right)$ is recommended to treat ID and iron deficiency anemia (IDA). ${ }^{3}$ Because iron absorption from oral supplements tends to be low, current recommendations call for daily provision of high doses of $\mathrm{FeSO}_{4}$, in the range of 60-200 $\mathrm{mg}$, preferably split into 2 or 3 daily doses..$^{4-7}$ With larger iron doses, ${ }^{8}$ the proportion of the dose absorbed, termed the fractional iron absorption (FIA), decreases, and large amounts of unabsorbed iron can cause gut inflammation $^{9,10}$ and increase free radical production and peroxidation in the gut mucosa. ${ }^{11,12}$ This may result in gastrointestinal side effects, which are common ${ }^{9}$ and typically dose dependent. ${ }^{13}$ Furthermore, an increase in colonic iron can reduce abundances of beneficial commensal gut bacteria and increase abundances of potential enteropathogens. ${ }^{14,15}$

Hepcidin, the central systemic controller of iron homeostasis in mammals is a 25 -amino acid peptide mainly produced by the liver, and is regulated by iron, hypoxia, inflammation and erythropoiesis. ${ }^{16}$ Hepcidin binds to ferroportin, mainly expressed on enterocytes, hepatocytes and macrophages, leading to internalization and degradation of ferroportin. Thus, high serum hepcidin (SHep) reduces dietary iron absorption and recycling of iron from senescent erythrocytes. Large oral doses of iron acutely increase SHep in a dose-dependent fashion, with the increase in SHep persisting for $\sim 24$ hours (h). 8,17 The increase after iron administration is distinct ${ }^{18}$ from the natural circadian increase in SHep over the day. ${ }^{19}$ We previously showed that twice daily administration of $60 \mathrm{mg}$ oral iron sharply augments the circadian SHep increase and results in higher SHep on the next day compared to once daily dosing with $120 \mathrm{mg}$ iron. ${ }^{17}$

In iron-depleted young women given doses $\geq 60 \mathrm{mg}$ of oral iron in the morning, SHep increases and is followed by a decrease in iron absorption on the following day. ${ }^{8}$ Consequently, alternate day dosing results in a higher FIA compared to daily dosing. ${ }^{8}$ In a study comparing iron absorption from $60 \mathrm{mg}$ doses during 28 days of alternate day versus 14 days of consecutive day supplementation, FIA was significantly higher (+33\%) with alternate day dosing. ${ }^{17}$ In addition, due to the acute SHep increase after an oral iron dose, splitting a dose into two daily divided doses did not increase iron absorption. ${ }^{17}$ However, these studies were conducted in iron-depleted women without anemia. Whether oral iron supplements, given at higher doses in women with IDA also induce an acute SHep increase and inhibit absorption of daily doses of iron is uncertain. $^{20}$

Besides the iron-induced increase in SHep, a putative 'mucosal block' may decrease iron absorption from daily iron doses. According to the 'mucosal block' theory, enterocytes exposed to a large dose of iron will not absorb subsequent iron doses until they are replaced by new enterocytes after five to six days; therefore, provision of iron doses at weekly intervals might increase absorption. ${ }^{21}$ If the increase in SHep subsides after $48 \mathrm{~h}$, any residual inhibition on absorption would be consistent with this view of the 'mucosal block'. The World Health Organisation recommends weekly intermittent iron doses in women who experience significant side effects taking oral iron doses..$^{22}$ Therefore, the aim of our study was to measure the magnitude and duration of the acute SHep increase after high-dose oral iron supplementation and the effect on iron absorption in women with IDA. Our hypotheses regarding women with IDA were: a) single oral iron doses of 100 and 200 mg acutely increase SHep and this increase persists for $24 \mathrm{~h}$, but not $48 \mathrm{~h}$; b) FIA from both doses would be lower on the following day, but not differ from baseline $48 \mathrm{~h}$ post administration (alternate day dosing), suggesting there is no 'mucosal block'; and c) FIA would be lower from the $200 \mathrm{mg}$ dose than the 100 mg dose.

\section{Methods}

\section{Subjects}

We recruited healthy women participating in the blood donation drive at the University of Zürich, and we conducted this study at the Human Nutrition Laboratory of the ETH Zürich, Switzerland. Detailed inclusion criteria are described in the Online Supplementary Materials and Methods. In this cross-over study, we compared iron absorption from consecutive and alternate day dosing in women with IDA, using 100 and $200 \mathrm{mg}$ doses of iron as ferrous sulfate $\left(\mathrm{FeSO}_{4}\right)$. This study was approved by the Cantonal Ethics Committee in Zürich, Switzerland. All participants gave informed written consent.

Participants went through two study cycles of 6 days each, with 16 days in between (Figure 1). To all subjects, we administered oral doses of $\mathrm{FeSO}_{4}$ in the morning on two consecutive days (days 2 and 3 ) and a third dose $48 \mathrm{~h}$ later (day 5), each dose was labeled with ${ }^{57} \mathrm{Fe}, 58 \mathrm{Fe}$ or ${ }^{54} \mathrm{Fe}$. Subjects were randomly assigned to first receive either three doses of $100 \mathrm{mg}$ or three doses of $200 \mathrm{mg}$. They were given the iron dose under standardized conditions. Detailed supplement administration is described in the Online Supplementary Material and Methods. On day 1, before iron supplementation, baseline venipuncture blood samples were taken at 8:00 AM and at 4:00 PM. Iron was administered at 8:00 AM on days 2, 3 and 5. Blood samples were taken at 8:00 AM (before dosing) and at 4:00 PM on days 2, 3 and 5. Additional blood samples were taken on day 4 and day 6 at 8:00 AM. Using a questionnaire, subjects were asked whether they had gastrointestinal side effects during the visits. We assessed iron absorption by measuring isotopic enrichment in red blood cells 16 days after administration of the third dose in both supplementation periods. ${ }^{23-25}$ Hemoglobin $(\mathrm{Hb})$, SHep, iron- and inflammatory biomarkers were measured as described in the Online Supplementary Materials and Methods.

Based on previous studies in our laboratory at the ETH Zürich using iron supplements in women we expected a standard deviation (SD) of the difference between pairs of 0.18 in log FIA. The study was powered to detect a relevant difference of $30 \%$ in FIA on a linear scale (such as $15 \%$ and $20 \%$ ), which on a log scale, corresponds to 0.125 units of $\log$ FIA. This yielded a minimal sample size of 18 subjects, assuming a power of 0.8 and an alpha of 0.05 . We recruited an additional subject to account for possible attrition, and enrolled 19 women in the study.

\section{Statistical analysis}

We performed the statistical analyses using SPSS (IBM SPSS statistics, Version22), as described in detail in the Online Supplementary Materials and Methods. We used linear mixed effect model analysis with Bonferroni corrected multiple comparisons to assess the effect of consecutive versus alternate day dosing with 100 and $200 \mathrm{mg}$ on different variables. Dose and time (reflecting the supplement 
administration day) were defined as fixed effects, participants as random effects (intercept) using a variance component structure matrix. Spearman correlation was applied. Incidences of side effects were compared using $\chi^{2}$ test. $P$-values $<0.05$ were considered statistically significant.

\section{Results}

We began recruiting on October 10, 2017, and during October and November 2017, we enrolled 19 women in the study. Three women were included in the study based on their $\mathrm{Hb}$ concentration before blood donation; 16 women were included in the study based on their $\mathrm{Hb}$ concentration after blood donation. We completed the study on January 8 , 2018. Three women left the study after completing the first supplementation period: two after the $100 \mathrm{mg}$ dosing (one because of gastrointestinal side effects, one because of travels away from the study site) and one after the $200 \mathrm{mg}$ dosing (because of travels away from the study site). The data from these three women from the first supplementation period were included in the final analysis.

\section{Baseline characteristics}

Table 1 shows the subject characteristics at baseline (day 1 ) and at day 22, by randomization group. At baseline, four subjects were borderline anemic with a baseline $\mathrm{Hb}$ between 12.0-12.5 g/dL (two in each group) and three subjects, who received $100 \mathrm{mg}$ iron first, had a mild upper respiratory tract infection and had an elevated baseline creactive protein (CRP); all of these subjects were included in the analysis. There were no significant within-group or

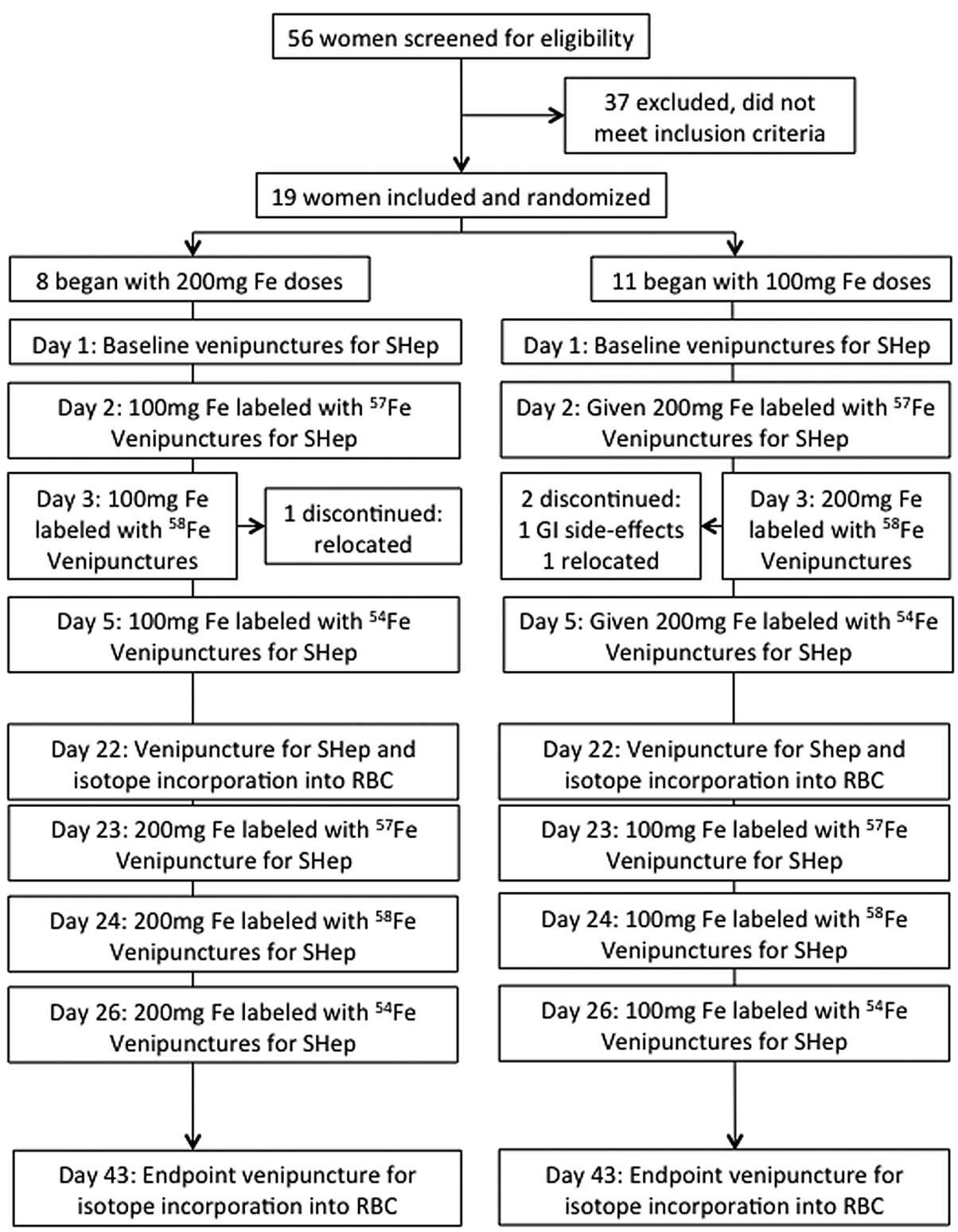

Figure 1. Study design. SHep: serum hepcidin; RBC: red blood cells. 
between-group differences in age, body mass index (BMI) or iron and inflammatory parameters at days 1 and 22 (Table 1).

\section{Hepcidin profiles and iron absorption during daily and intermittent oral iron dosing}

As shown in Figure 2, median (IOR) SHep (nM) at 8 AM before administration of the $100 \mathrm{mg}$ dose on days 2, 3 and 5 was 0.24 (0.19-0.38), 0.60 (0.44 - 1.09) and 0.46 (0.20-0.62), and at $8 \mathrm{AM}$ before administration of the 200 mg dose on days 2,3 and 5 was 0.26 (0.13-0.37), 0.74 (0.38-1.62) and $0.42(0.29-0.74)$. SHep was significantly affected by time $(P<0.001)$ but not by dose $(P=0.733)$, and there was no significant time-dose interaction $(P=0.815)$. For both doses, SHep was significantly higher on day 3 compared to day $2(P<0.001)$ or day $5(P<0.01)$. There was no significant difference in SHep between days 2 and $5(P=0.115)$. Individual SHep data for each participant on days 2, 3 and 5 for both doses are shown in the Online Supplementary Figure S1.

Summing the 3 doses from each supplementation period, there was a significant dose effect (100 vs. $200 \mathrm{mg}$ iron) on both FIA and total iron absorption (TIA) $(P<0.001$ for both), but no significant time-dose interaction on FIA or TIA $(P=0.737 ; P=0.763)$. For both doses, there was a significant time effect on FIA (Figure $3 \mathrm{~A}-\mathrm{B}$ ) and TIA $(P<0.001$ for both). Geometric mean $(-\mathrm{SD},+\mathrm{SD})$ FIA $(\%)$ from the $100 \mathrm{mg}$ dose on days 2,3 and 5 was 23.5 (17.2, $32.2), 17.0(12.3,23.6)$ and $25.0(16.8,37.2)$, respectively,

Table 1. Characteristics of the women $(n=19)$ at day 1 (baseline, before beginning the first set of iron doses) and at day 22 (before beginning the second set of iron doses), by group.

$\begin{array}{cc}\text { Group first } & \text { Group first } \\ \text { receiving } & \text { receiving } \\ 100 \mathrm{mg} & 200 \mathrm{mg} \\ \text { iron doses } & \text { iron doses }\end{array}$

\begin{tabular}{lcc} 
& \multicolumn{2}{c}{ Day 1} \\
Age, $\mathrm{y}^{1}$ & $21(19-24)$ & $22(20-26)$ \\
\hline Body mass index, $\mathrm{kg} / \mathrm{m}^{2}$ & $22.7 \pm 2.3$ & $21.6 \pm 2.2$ \\
Hemoglobin, $\mathrm{g} / \mathrm{dL}$ & $11.3 \pm 1.1$ & $11.6 \pm 0.6$ \\
\hline Serum ferritin, $\mu \mathrm{g} / \mathrm{L}$ & $9.6 \pm 4.3$ & $12.0 \pm 4.7$ \\
Serum sTfR, $\mathrm{mg} / \mathrm{L}$ & $5.2(4.5-6.7)$ & $5.2(4.5-6.3)$ \\
\hline Serum hepcidin, $\mathrm{nM}$ & $0.32(0.16-0.44)$ & $0.19(0.14-0.44)$ \\
Serum C-reactive protein, mg/L & $0.50(0.34-8.35)$ & $0.37(0.13-4.0)$ \\
\hline Serum alpha glycoprotein, g/L & $0.47(0.38-0.59)$ & $0.49(0.32-0.62)$ \\
& \multicolumn{2}{c}{ Day $\mathbf{2 2}$}
\end{tabular}

\begin{tabular}{lcc} 
Hemoglobin, $\mathrm{g} / \mathrm{dL}$ & $12.1 \pm 0.7$ & $12.0 \pm 1.2$ \\
\hline Serum ferritin, $\mu \mathrm{g} / \mathrm{L}$ & $9.4 \pm 3.4$ & $8.1 \pm 3.9$ \\
Serum sTfR, $\mathrm{mg} / \mathrm{L}$ & $5.9(5.4-8.8)$ & $6.7(4.5-8.4)$ \\
\hline Serum hepcidin, $\mathrm{nM}$ & $0.10(0.06-0.27)$ & $0.16(0.02-0.22)$ \\
Serum C-reactive protein, $\mathrm{mg} / \mathrm{L}$ & $0.25(0.16-2.14)$ & $1.23(0.30-2.75)$ \\
\hline Serum alpha glycoprotein, $\mathrm{g} / \mathrm{L}$ & $0.54(0.36-1.21)$ & $0.47(0.40-0.79)$ \\
\hline
\end{tabular}

'All such data as medians (IQR). ${ }^{2}$ All such data as means \pm standard deviation (SD). sTFR=soluble transferrin receptor. There were no significant differences between groups at day 1 and day 22 , as well as no significant differences within groups comparing days 1 and 22. For between group comparisons, independent sample t-test was used for normally distributed data and Mann-Whitney U test was used for not normally distributed data. For within group comparisons, dependent sample t-test was used for normally distributed data and Wilcoxon matched-pair signed-rank test was used for not normally distributed data. and from the $200 \mathrm{mg}$ dose on days 2,3 and 5 was 17.3 (12.1, 24.8), $11.9(8.5,16.6)$ and $16.8(10.8,25.9)$, respectively. FIA and TIA on day 2 and day 5 was significantly higher than on day $3(P<0.001)$, but did not differ significantly comparing days 2 and 5 , independent of the dose (Figure 3). Individual FIA data for each participant on days 2,3 and 5 for both doses are shown in the Online Supplementary Figure S2. FIA from daily dosing (day 3) with $100 \mathrm{mg}$ was not significantly different from alternate dosing (day 5) with $200 \mathrm{mg}(P=0.792)$, but TIA was greater from the $200 \mathrm{mg}$ dose on the alternate day $(P<0.001)$. There was no significant correlation between baseline $\mathrm{Hb}$ and the difference in FIA between alternate (day 5) and consecutive (day 3) dosing $\left(r_{s}=-0.292\right.$; $P=0.240)$

\section{The effects of high oral iron doses on iron- and inflammatory status}

Iron and inflammatory status indicators are shown in Table 2. There was a significant dose (100 vs. $200 \mathrm{mg}$ iron) and time effect on serum ferritin (SF) $(P<0.01, P<0.001)$. For both doses, SF on days 3 and 5 was significantly higher than on day $2(P<0.001)$ and SF was also significantly higher on day 3 than on day $5(P<0.05)$. There were significant time effects on serum iron (SFe), total iron binding capacity (TIBC) and transferrin saturation (TSAT) $(P<0.05$ for all). SFe and TSAT on days 3 and 5 were significantly higher than on day $2(P<0.05$ for all), but did not differ signifi cantly comparing days 3 and 5 , independent of the dose. TIBC on days 3 and 5 was significantly lower than on day $2(P<0.05, P<0.01)$, but did not differ significantly comparing days 3 and 5 , for both doses. The iron doses had no significant effect on erythropoietin (EPO), and there was no significant difference

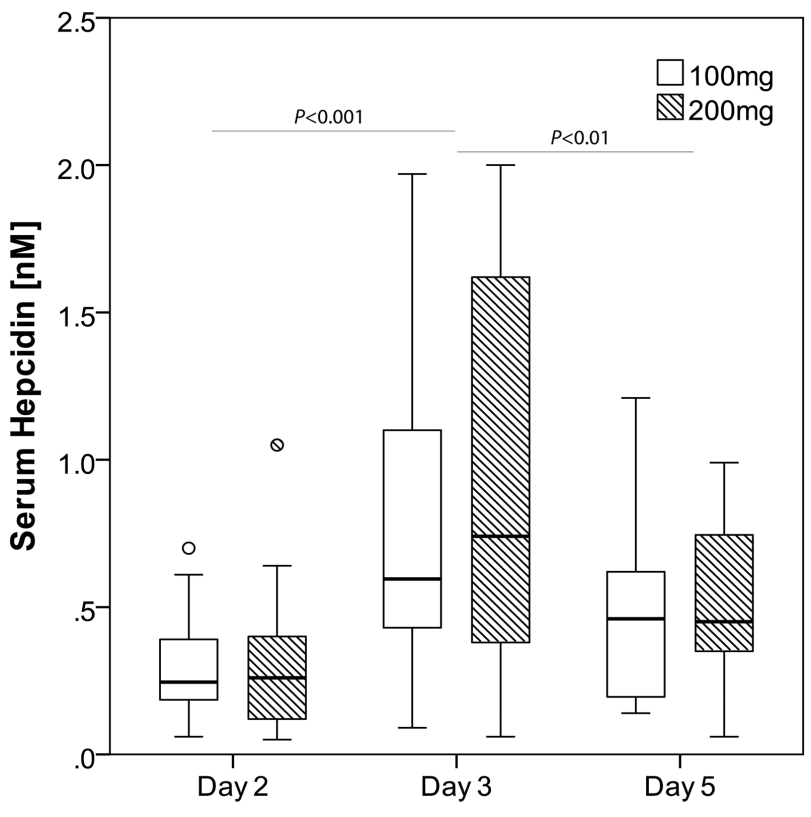

Figure 2. Serum hepcidin in iron-deficient anemic women. In women with iron deficiency anemia (IDA) who received $100 \mathrm{mg}$ and $200 \mathrm{mg}$ oral iron supplements on consecutive (day 3 ) and alternate days (day 5), serum hepcidin increases at 24 hours $(\mathrm{h})$ and returns to baseline by $48 \mathrm{~h}$. 
Table 2. Iron and inflammatory indices in iron-deficient anemic women ( $\mathrm{n}=19)$ receiving 100 or $200 \mathrm{mg}$ of oral iron as $\mathrm{FeSO}_{4}$ on days 2,3 and 5.

\begin{tabular}{|c|c|c|c|c|c|c|c|c|}
\hline & \multicolumn{3}{|c|}{100 mg iron supplement } & \multicolumn{3}{|c|}{200 mg iron supplement } & \multicolumn{2}{|c|}{$\boldsymbol{P}$} \\
\hline & Day 2 & Day 3 & Day 5 & Day 2 & Day 3 & Day 5 & Dose & Time (day) \\
\hline Serum ferritin, $\mu g / \mathrm{L}^{1}$ & $\begin{array}{c}9.62 \\
(5.75,16.09)\end{array}$ & $\begin{array}{c}18.19 \\
(10.93,30.26)\end{array}$ & $\begin{array}{c}14.35 \\
(7.73,26.67)\end{array}$ & $\begin{array}{c}10.02 \\
(6.59,15.26)\end{array}$ & $\begin{array}{c}21.99 \\
(13.79,35.05)\end{array}$ & $\begin{array}{c}18.46 \\
(10.37,32.84)\end{array}$ & $<0.01$ & $<0.001$ \\
\hline Serum sTfR, mg/L $\mathrm{L}^{2}$ & $\begin{array}{c}6.10 \\
(5.24-7.16)\end{array}$ & $\begin{array}{c}5.67 \\
(4.88-6.29)\end{array}$ & $\begin{array}{c}5.82 \\
(4.69-6.91)\end{array}$ & $\begin{array}{c}6.35 \\
(4.92-7.49)\end{array}$ & $\begin{array}{c}5.16 \\
(4.71-6.66)\end{array}$ & $\begin{array}{c}5.58 \\
(4.51-6.25)\end{array}$ & 0.413 & $<0.001$ \\
\hline Serum iron, $\mu \mathrm{M}$ & $\begin{array}{c}6.87 \\
(3.90,12.10)\end{array}$ & $\begin{array}{c}11.26 \\
(6.56,19.33)\end{array}$ & $\begin{array}{c}9.74 \\
(5.97,15.88)\end{array}$ & $\begin{array}{c}7.69 \\
(4.45,13.29)\end{array}$ & $\begin{array}{c}12.09 \\
(5.65,25.85)\end{array}$ & $\begin{array}{c}12.44 \\
(8.08,19.14)\end{array}$ & 0.061 & $<0.001$ \\
\hline TIBC, $\mu \mathrm{M}$ & $\begin{array}{c}86.68 \\
(74.64,100.66)\end{array}$ & $\begin{array}{c}81.49 \\
(73.91,89.84)\end{array}$ & $\begin{array}{c}82.00 \\
(72.45,92.81)\end{array}$ & $\begin{array}{c}91.71 \\
(80.96,103.90)\end{array}$ & $\begin{array}{c}84.38 \\
(73.66,96.66)\end{array}$ & $\begin{array}{c}83.12 \\
(72.93,94.74)\end{array}$ & 0.268 & $<0.01$ \\
\hline TSAT, $\%$ & $\begin{array}{c}7.92 \\
(4.49,13.99)\end{array}$ & $\begin{array}{c}13.82 \\
(8.18,23.37)\end{array}$ & $\begin{array}{c}11.87 \\
(7.00,20.13)\end{array}$ & $\begin{array}{c}8.61 \\
(4.87,15.21)\end{array}$ & $\begin{array}{c}15.68 \\
(7.96,30.90)\end{array}$ & $\begin{array}{c}15.06 \\
(9.41,24.10)\end{array}$ & 0.106 & $<0.001$ \\
\hline Serum EPO, mIU/mL & $\begin{array}{c}15.35 \\
(8.60,27.38)\end{array}$ & $\begin{array}{c}15.09 \\
(8.09,28.13)\end{array}$ & $\begin{array}{c}17.02 \\
(9.95,29.12)\end{array}$ & $\begin{array}{c}15.12 \\
(7.87,29.04)\end{array}$ & $\begin{array}{c}18.21 \\
(9.67,34.29)\end{array}$ & $\begin{array}{c}16.43 \\
(8.74,30.86)\end{array}$ & 0.727 & 0.590 \\
\hline CRP, mg/L & $\begin{array}{c}0.53 \\
(0.24-2.83)\end{array}$ & $\begin{array}{c}0.52 \\
(0.21-3.21)\end{array}$ & $\begin{array}{c}0.38 \\
(0.25-1.20)\end{array}$ & $\begin{array}{c}0.89 \\
(0.43-2.12)\end{array}$ & $\begin{array}{c}0.78 \\
(0.31,2.84)\end{array}$ & $\begin{array}{c}0.46 \\
(0.23,1.97)\end{array}$ & 0.556 & 0.072 \\
\hline AGP, g/L & $\begin{array}{c}0.54 \\
(0.32,0.91)\end{array}$ & $\begin{array}{c}0.56 \\
(0.35,0.91)\end{array}$ & $\begin{array}{c}0.46 \\
(0.29,0.72)\end{array}$ & $\begin{array}{c}0.57 \\
(0.36,0.90)\end{array}$ & $\begin{array}{c}0.46 \\
(0.23,0.93)\end{array}$ & $\begin{array}{c}0.31 \\
(0.16,0.62)\end{array}$ & 0.101 & $<0.01$ \\
\hline
\end{tabular}

'All such data as geometric means (-SD,+SD). ${ }^{2}$ All such data as medians (IQR). Analyzed by Linear Mixed Model Analysis with Bonferroni corrected multiple comparisons. There were no significant dose by time interactions. sTFR: soluble transferrin receptor; TIBC: total iron binding capacity; TSAT: transferrin saturation; EPO: erythropoietin; CRP: C-reactive protein; AGP: alpha-1-acid glycoprotein.

in EPO between the two doses on any of the study days (Table 2).

The increases in SHep and TSAT in the afternoon after oral iron administration (Figure 4A-B) reflected the dose. Linear mixed model analysis showed significant time and daytime effects $(P<0.001$ for both), but no dose effect $(P=0.168)$ on SHep. There was a significant time by daytime interaction $(P<0.001)$, but there were no dose by time, dose by daytime or dose by time by daytime interactions $(P=0.981, \mathrm{P}=0.390, P=0.940)$. Linear mixed model analysis showed significant dose, time and daytime effects $(P<0.001$ for all), on TSAT. There were significant dose by daytime and time by daytime interactions $(P<0.01, P<0.001)$, but there were no dose by time or dose by time by daytime interactions $(P=0.265, P=0.185)$.

\section{Gastrointestinal side effects}

All reported adverse events were grade I-II. The most common adverse event was nausea/epigastric pain for both doses: five cases in five women during $100 \mathrm{mg}$ dosing and 12 cases in eight women during $200 \mathrm{mg}$ dosing. There were three cases of vomiting $\sim 5 \mathrm{~h}$ after the iron intake: one after the first $100 \mathrm{mg}$ dose and two after the first $200 \mathrm{mg}$ iron dose. The total incidence of the gastrointestinal side effects that were assessed (epigastric pain/nausea/diarrhea/vomiting) was $40 \%$ lower with 100 $\mathrm{mg}$ dosing than with $200 \mathrm{mg}$ dosing, however this difference was not statistically significant $(P=0.105)$.

\section{Discussion}

Our main findings in women with IDA are: a) single oral iron doses of 100 and 200 mg acutely increased SHep and these increases persisted for $24 \mathrm{~h}$; b) FIA from both iron doses was lower with consecutive versus alternate day dosing; and c) TIA was higher from the $200 \mathrm{mg}$ dose than the $100 \mathrm{mg}$ dose while FIA was lower; d) with both doses, we did not detect a decrease in iron absorption after $48 \mathrm{~h}$ from the last dose, evidence against the postulate of a mucosal block lasting up to five or six days.

In this study, for both iron doses, SHep was significantly higher with consecutive day doses (on day 3 compared to day $2, P<0.01)$ and significantly lower with alternate day doses (on day 5 compared to day $3, P<0.05$ ), with no significant difference in SHep between days 2 and 5 . These data support our previous oral iron supplementation studies in iron-deficient, mostly non-anemic, subjects. ${ }^{8,17}$ In those studies, we assessed the magnitude and duration of the SHep increase after an oral iron dose and found that oral iron doses $\geq 60 \mathrm{mg}$ significantly increased SHep at $24 \mathrm{~h}$, which returned to baseline by $48 \mathrm{~h} .{ }^{8}$ In a second study, we provided 14 oral iron doses of $60 \mathrm{mg}$ to iron-depleted women either on 14 consecutive days or on alternate days over 28 days and showed that during the first 14 days of supplementation in both groups, SHep was higher in the consecutive day group than in the alternate day group, likely driven by the higher iron supplement frequency in the consecutive day group. ${ }^{17}$ Therefore, taken together, the available data suggest that in iron-deficient women with or without anemia, high oral iron doses acutely increase SHep and that this increase persists for $24 \mathrm{~h}$ but returns to baseline by $48 \mathrm{~h}$.

In this study, alternate day dosing resulted in a significantly higher FIA (by 40-50\%) compared to daily dosing for both 100 and $200 \mathrm{mg}$ doses $(P<0.001)$. FIA was significantly lower on day 3 compared to day $2(P<0.001)$ and significantly greater on day 5 compared to day 3 $(P<0.001)$ with no significant difference in FIA between days 2 and 5. Again, these results are consistent with those previously shown in iron-depleted, non-anemic women, where FIA was lower from an oral iron dose $\geq 60$ $\mathrm{mg}$ given the next day (at $24 \mathrm{~h}$ after a first dose) (8). Over 14 oral iron doses given either on 14 (consecutive) or 28 

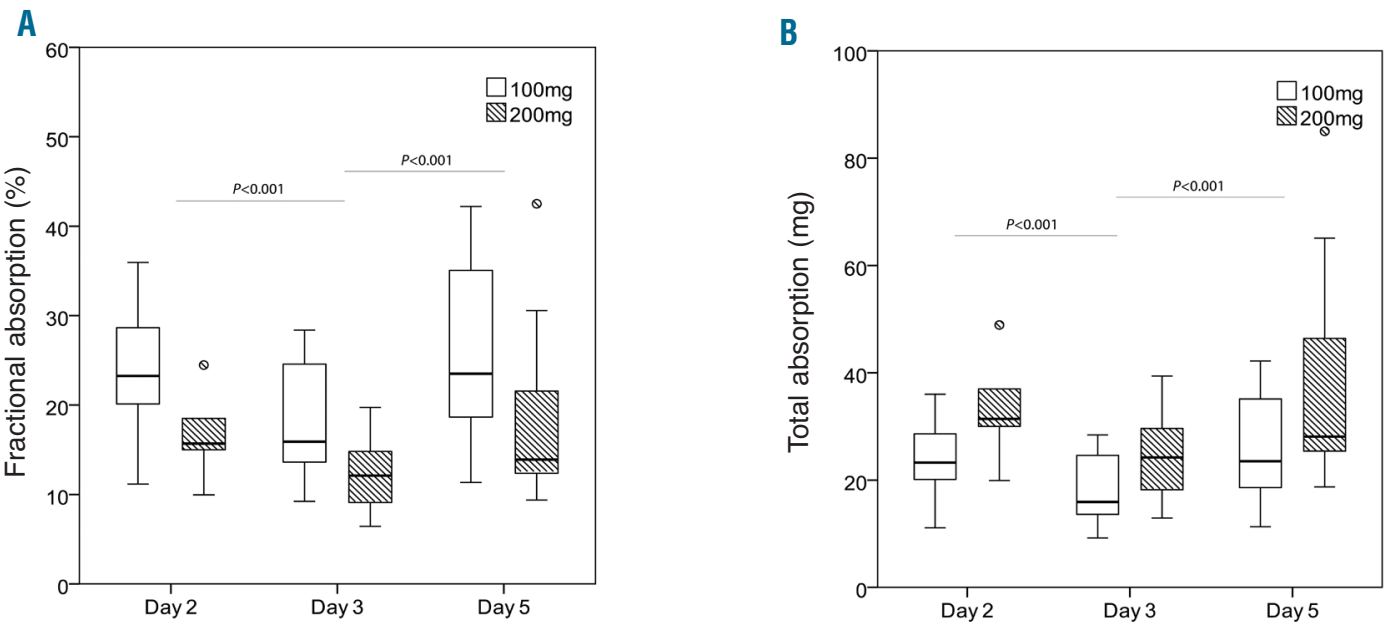

Figure 3. Iron absorption in iron-deficient anemic women. (A) Fractional iron absorption from 100 and 200 mg oral iron doses was higher during alternate day dosing (day 5) compared to consecutive day dosing (day 3); (B) Total iron absorption from 100 and $200 \mathrm{mg}$ oral iron doses was higher during alternate day dosing (day 5) compared to consecutive day dosing (day 3 ).
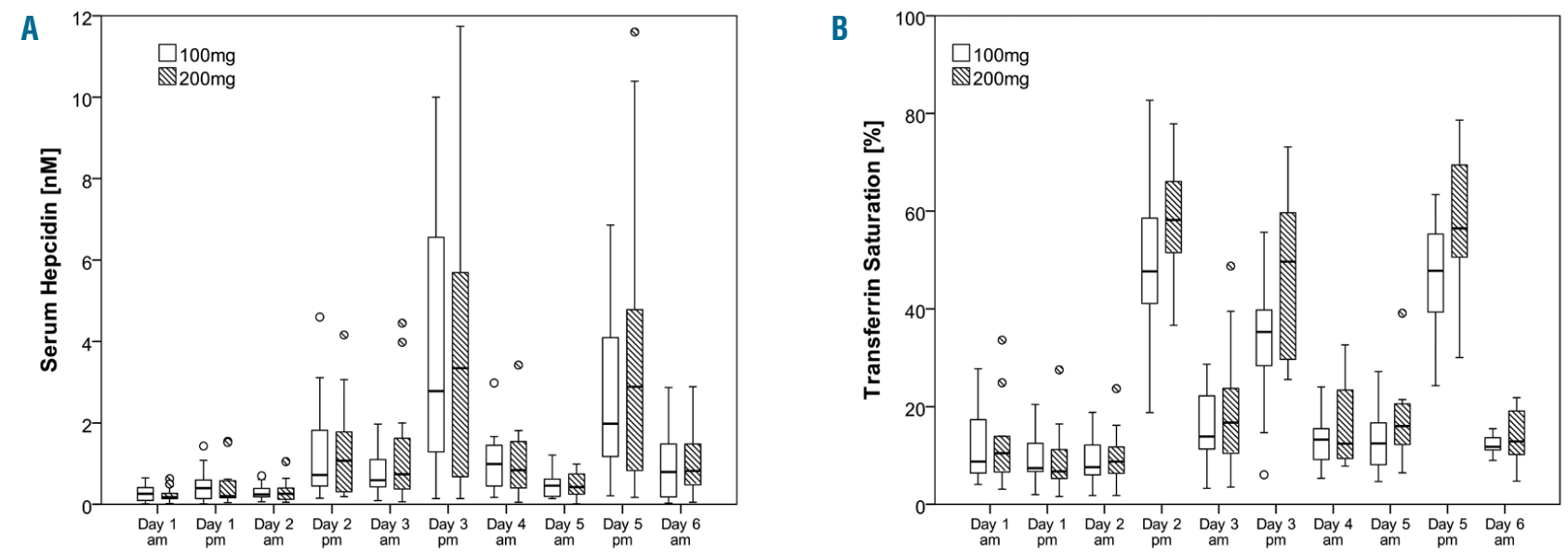

Figure 4. Serum hepcidin and transferrin saturation profiles in iron-deficient anemic women. (A) Serum hepcidin (Shep) profile during alternate day dosing (day 5 ) and consecutive day dosing (day 3) with 100 and 200 mg oral iron; (B) Transferrin saturation profile (TSAT) during alternate day dosing (day 5) and consecutive day dosing (day 3) with 100 and $200 \mathrm{mg}$ oral iron.

(alternate) days, alternate day dosing resulted in a $34 \%$ higher FIA compared to consecutive day dosing. ${ }^{17}$ In the current study, there was no residual absorption inhibition at $48 \mathrm{~h}$ post-administration; this is evidence against the postulate of a mucosal block lasting up to 5 or 6 days. ${ }^{26}$ This finding suggests that increasing the dosing interval beyond $48 \mathrm{~h}$ would not result in a further increase in iron absorption. Taken together, the available data suggest that in iron-deficient women with or without anemia, alternate day dosing with oral iron doses in the range of 60 to $200 \mathrm{mg}$ results in a sharply higher FIA compared to daily dosing.

In this study, FIA from $200 \mathrm{mg}$ was significantly lower compared to FIA from $100 \mathrm{mg}$ iron $(P<0.001)$. Thus, even in iron-deficient anemic women, who are maximizing enterocyte iron uptake from the gut lumen by upregulation of divalent metal transporter 1 (DMT1) expression, ${ }^{27}$ and who are maximizing enterocyte iron transfer to the circulation via low baseline SHep and high ferroportin expression, low oral iron doses are more efficiently absorbed than higher doses. Previous studies have generally demonstrated that FIA of oral iron decreases with increasing dose, but many of these studies were done in non-anemic subjects. ${ }^{8}$ In this study, there was no significant difference in FIA comparing daily dosing (day 3) with $100 \mathrm{mg}$ versus alternate day dosing with $200 \mathrm{mg}$ (day 5). Consequently, TIA from a single dose of $200 \mathrm{mg}$ given on alternate days was approximately twice that from 100 mg given on consecutive days $(P<0.001)$. This suggests that TIA would be similar from alternate day dosing of $200 \mathrm{mg}$ compared to daily dosing of $100 \mathrm{mg}$.

In women with IDA, the SHep increase of $\sim 0.4 \mathrm{nM}$ after doses of 100 and $200 \mathrm{mg}$ was much smaller than the SHep increase of $\sim 2 \mathrm{nM}$ (about $1.85 \mathrm{nM}$ after correction for method comparison) ${ }^{28}$ after doses of $120 \mathrm{mg}$ in nonanemic iron depleted women. ${ }^{8}$ A potential explanation 
for the lower increase in SHep in response to oral iron in anemic women compared to iron deficient non-anemic women is that hepatic hepcidin expression is more strongly suppressed by ID and erythropoetic drive; the latter mechanism may be particularly important in our subjects who had recently donated blood. ${ }^{16}$

The strengths of this study are that we used a crossover design providing two high oral iron doses (100 and $200 \mathrm{mg}$ ) to women with IDA together with a standardized diet, and precisely quantified iron absorption using multiple iron stable isotopic labels on multiple days, with each subject acting as her own control. SHep and iron status parameter profiles were accurately and repeatedly quantified using an immunoassay with high sensitivity over two to six days; tolerability and gastrointestinal side effects were assessed by a standardized interview. Furthermore, study participants were otherwise healthy and free of comorbidities. Limitations of the study are that our subjects were only mildly, or for some, borderline, anemic, with $\mathrm{Hb}$ values ranging from 8.6 to 12.5 $\mathrm{g} / \mathrm{dL}$. Confirmation of these findings in women with more severe anemia (with $\mathrm{Hb}<8 \mathrm{~g} / \mathrm{dL}$ ) would require further study. At inclusion, most of the participants had just donated blood, which contributed to their anemia. The recent loss of $500 \mathrm{~mL}$ blood during donation could have influenced the response to the supplemental iron doses: for example, acute blood loss can stimulate renal EPO production which can suppress hepatic hepcidin synthesis. ${ }^{29,30}$ However, despite prevailing high EPO concentrations (Table 2), we saw clear increases in SHep in response to the iron doses. Whether the response of SHep would differ in subjects with chronic anemia cannot be concluded from our data. However, chronic anemia would also increase the chance for gastrointestinal or other inflammatory conditions affecting iron absorption, and we can exclude that these had an effect in the current study. Finally, based on our study design, it is unclear if the effect of iron supplement dosing on consecutive days (day 2, 3) prior to the alternate day dosing (day 3, 5) effected our comparisons. To address this, an alternate design could have been to test consecutive day dosing and then have a washout period and then test the alternate day dosing. However, a disadvantage of this approach would be an increase in the number of test meals and subject burden, as well as potential changes in subjects' inflammatory and/or iron status during the washout period that would increase variability.

In conclusion, as in our previous studies using a daily dose of $60 \mathrm{mg}$ in iron-depleted non-anemic women, our data show that with higher doses of 100 to $200 \mathrm{mg}$ iron in women with IDA, alternate day dosing results in higher FIA and a trend for lower incidence of gastrointestinal side effects compared to consecutive day dosing. These potential benefits need to be confirmed in long-term intervention studies in anemic women with clinical endpoints, such as change in $\mathrm{Hb}$, iron status and side effects, as primary outcomes. If confirmed, this dosing regimen may allow the use of lower iron doses, which may reduce side effects and improve compliance.

\section{Acknowledgments}

The authors thank the participating women, the nursing staff, Bérénice Hansen, Dr. Reinhard Henschler and Dr. Beat Frey for their support of the study.

\section{References}

1. Kassebaum NJ, Jasrasaria R, Naghavi M, et al. A systematic analysis of global anemia burden from 1990 to 2010. Blood. 2014; 123(5):615-624.

2. Cogswell ME, Looker AC, Pfeiffer CM, et al. Assessment of iron deficiency in US preschool children and nonpregnant females of childbearing age: National Health and Nutrition Examination Survey 2003-2006. Am J Clin Nutr. 2009;89(5):1334-1342.

3. Cook JD. Diagnosis and management of iron-deficiency anaemia. Best Pract Res Clin Haematol. 2005;18(2):319-332.

4. Camaschella C. Iron deficiency: new insights into diagnosis and treatment. Hematology Am Soc Hematol Educ Program. 2015;2015:8-13.

5. CDC. Recommendations to Prevent and Control Iron Deficiency in the United States. Atlanta: Centers for Disease Control and Prevention (CDC). 1998 [cited 2018 21.09]; Available from: https://www.cdc.gov/mmwr/preview/mm wrhtml/00051880.htm

6. Stoltzfus RJ, Dreyfuss ML. Guidelines for the use of iron supplements to prevent and treat iron deficiency anemia: International Nutritional Anemia Consultative Group (INACG), 1998. Available from: motherchildnutrition.org/nutrition-protection-promotion/pdf/mcn-guidelines-for-iron-supplementation.pdf

7. Pavord S, Myers B, Robinson S, et al. UK guidelines on the management of iron deficiency in pregnancy. Br J Haematol. 2012; 156(5):588-600

8. Moretti D, Goede JS, Zeder C, et al. Oral iron supplements increase hepcidin and decrease iron absorption from daily or twice-daily doses in iron-depleted young women. Blood. 2015;22;126(17):19811989.

9. Tolkien Z, Stecher L, Mander AP, Pereira DI, Powell JJ. Ferrous sulfate supplementation causes significant gastrointestinal sideeffects in adults: a systematic review and meta-analysis. PloS One. 2015;10(2): e0117383.

10. Kortman GA, Raffatellu M, Swinkels DW, Tjalsma H. Nutritional iron turned inside out: intestinal stress from a gut microbial perspective. FEMS Microbiol Rev 2014; 38(6):1202-1234

11. Lund EK, Fairweather-Tait SJ, Wharf SG, Johnson IT. Chronic exposure to high levels of dietary iron fortification increases lipid peroxidation in the mucosa of the rat large intestine. J Nutr. 2001;131(11):2928-2931.

12. Lund EK, Wharf SG, Fairweather-Tait SJ, Johnson IT. Oral ferrous sulfate supplements increase the free radical-generating capacity of feces from healthy volunteers. Am J Clin Nutr. 1999;69(2):250-255.

13. Rimon E, Kagansky N, Kagansky M, et al. Are we giving too much iron? Low-dose iron therapy is effective in octogenarians. Am J Med. 2005;118(10):1142-1147.

14. Jaeggi T, Kortman GA, Moretti D, et al
Iron fortification adversely affects the gut microbiome, increases pathogen abundance and induces intestinal inflammation in Kenyan infants. Gut. 2015;64(5):731-742.

15. Paganini D, Uyoga MA, Kortman GAM, et al. Prebiotic galacto-oligosaccharides mitigate the adverse effects of iron fortification on the gut microbiome: a randomised controlled study in Kenyan infants. Gut. 2017;66(11):1956-1967.

16. Sangkhae V, Nemeth E. Regulation of the iron homeostatic hormone hepcidin. Adv Nutr. 2017;8(1):126-136.

17. Stoffel NU, Cercamondi CI, Brittenham G, et al. Iron absorption from oral iron supplements given on consecutive versus alternate days and as single morning doses versus twice-daily split dosing in iron-depleted women: two open-label, randomised controlled trials. Lancet Haematol. 2017;4(11): e524-e533.

18. Schaap CC, Hendriks JC, Kortman GA, et al. Diurnal rhythm rather than dietary iron mediates daily hepcidin variations. Clin Chem. 2013;59(3):527-535.

19. Troutt JS, Rudling M, Persson L, et al Circulating human hepcidin-25 concentrations display a diurnal rhythm, increase with prolonged fasting, and are reduced by growth hormone administration. Clin Chem. 2012;58(8):1225-1232.

20. Camaschella C. New insights into iron deficiency and iron deficiency anemia. Blood Rev. 2017;31(4):225-233.

21. Pena-Rosas JP, De-Regil LM, Gomez 
Malave H, Flores-Urrutia MC, Dowswell T. Intermittent oral iron supplementation during pregnancy. Cochrane DB Syst Rev. 2015 19(10):CD009997.

22. Pena-Rosas JP, Nesheim MC, Garcia-Casal $\mathrm{MN}$, et al. Intermittent iron supplementation regimens are able to maintain safe maternal hemoglobin concentrations during pregnancy in Venezuela. J Nutr. 2004; 134(5):1099-1104

23. Hotz K, Krayenbuehl PA, Walczyk T. Mobilization of storage iron is reflected in the iron isotopic composition of blood in humans. J Biol Inorg Chem. 2012;17(2):301309.

24. Brown E, Bradley B, Wennesland R, Hodges JL, Hopper J, Yamauchi H. Red
Cell, Plasma, and Blood Volume in Healthy Women Measured by Radichromium CellLabeling and Hematocrit. J Clin Invest. 1962;41:2182-2190

25. Hosain F, Marsaglia G, Noyes W, Finch CA. The nature of internal iron exchange in man. Trans Assoc Am Physicians. 1962; 75:59-63.

26. Hallberg L. Combating iron deficiency: daily administration of iron is far superior to weekly administration. Am J Clin Nutr. 1998;68(2):213-217.

27. Zoller H, Koch RO, Theurl I, et al. Expression of the duodenal iron transporters divalent-metal transporter 1 and ferroportin 1 in iron deficiency and iron overload. Gastroenterology. 2001;120(6):
1412-1419

28. Stoffel NU, Zeder C, Fort E, Swinkels DW, Zimmermann MB, Moretti D. Prediction of human iron bioavailability using rapid cELISAs for human plasma hepcidin. Clin Chem Lab Med. 2017;26;55(8):1186-1192.

29. Maeda H, Hitomi Y, Hirata R, et al. The effect of phlebotomy on serum erythropoietin levels in normal healthy subjects. Int Hematol. 1992;55(2):111-115.

30. Schotten N, Laarakkers CM, Roelofs RW Origa $R$, van Kraaij MG, Swinkels DW. $\mathrm{EPO}$ and hepcidin plasma concentrations in blood donors and beta-thalassemia intermedia are not related to commercially tested plasma ERFE concentrations. Am J Hematol. 2017;92(3):E29-E31. 